\title{
Ökonomische Aspekte in der klinischen Praxis aus der Perspektive onkologisch tätiger Ärzte in Deutschland und England: Ergebnisse einer explorativen Interviewstudie
}

\author{
Jan Schildmann ${ }^{\mathrm{a}}$ Jacinta $\operatorname{Tan}^{\mathrm{b}}$ Jochen Vollmann ${ }^{\mathrm{a}}$ \\ a Institut für Medizinische Ethik und Geschichte der Medizin, Ruhr-Universität Bochum, Germany \\ ${ }^{\mathrm{b}}$ Department of Philosophy, Law and Humanities, School of Health and Human Science, Swansea University, UK
}

\section{Schlüsselwörter}

Ressourcenallokation · Rationierung · Onkologie .

Qualitative Studien · Empirische Medizinethik

\section{Zusammenfassung}

Hintergrund: Die Kosten für die medizinische Versorgung und Strategien zum Umgang mit begrenzten Ressourcen im Gesundheitswesen sind Gegenstand der aktuellen Diskussion in vielen europäischen Ländern. Bislang liegen wenige empirische Untersuchungen zur Wahrnehmung und Bewertung ökonomischer Aspekte in der klinischen Praxis aus der Perspektive onkologisch tätiger Ärzte vor. Studienteilnehmer und Methoden: Semistrukturierte Leitfadeninterviews mit onkologisch tätigen Ärzten in Deutschland und England. Für diesen Beitrag wurden Transkript-Abschnitte, in denen die Befragten ökonomische Aspekte in der klinischen Praxis thematisierten, explorativ und nach Prinzipien der Inhaltsanalyse ausgewertet. Ergebnisse: Es wurden 17 (Deutschland) beziehungsweise 12 (England) Forschungsinterviews mit onkologisch tätigen Ärzten durchgeführt. Die in Deutschland tätigen Ärzte beschreiben verschiedene Formen der impliziten Rationierung. Die Auswirkungen ökonomischer Erwägungen werden überwiegend negativ bewertet. Die in England befragten Ärzte benennen konkrete Situationen, in denen Interventionen aus Kostengründen vorenthalten werden. Eine Rationierung medizinischer Maßnahmen wird angesichts begrenzter Ressourcen als notwendig erachtet. Die Zuordnung von Allokationsentscheidungen auf die Makroebene wird unter anderem aufgrund der daraus resultierenden Entlastung der Arzt-Patient-Beziehung befürwortet. Schlussfolgerungen: Die Narrative der Befragten aus den beiden Ländern unterscheiden sich hinsichtlich der Wahrnehmung und Bewertung ökonomischer Aspekte in der klinischen Praxis. Der jeweilige Umgang mit begrenzten Ressourcen in den beiden Gesundheitssystemen bietet einen möglichen Erklärungsansatz für diese Differenzen.

\author{
Keywords \\ Allocation of resources · Rationing - Oncology . \\ Qualitative studies · Empirical medical ethics
}

\section{Summary}

Economical Aspects in Clinical Practice: Results from an Explorative Interview Study on the Perspectives of Oncologists in Germany and England

Background: Costs for medical care and strategies to handle limited resources in health care are discussed in many European countries. Few empirical studies explore the oncologists' perceptions and views regarding economical aspects in clinical practice. Research Participants and Methods: Semi-structured interviews with physicians working in oncology in Germany and England. For this paper, parts of the transcripts in which interviewees discuss economical aspects of clinical practice were analysed exploratively and according to principles of content analysis. Results: 17 (Germany) and 12 (England) research interviews were analysed. German physicians described different forms of implicit rationing. The effects of economic considerations are judged as predominantly negative. Physicians working in England named concrete situations in which interventions are not performed because of cost considerations. The rationing of medical care was viewed as necessary in light of limited resources. The assignment of allocation decisions on the macro-level was favoured because of the resulting relief for the physician-patient relationship. Conclusions: The narratives of interviewees in both countries differ regarding the perceptions and views on economical aspects in clinical practice. The specific approaches of each health care system towards the allocation of limited resources offer one basis for explanations of these differences.

\begin{tabular}{ll}
\hline KARGER & ( ) 2011 S. Karger GmbH, Freiburg \\
0378-584X/11/3413-0020 \$38.00/0 \\
$\begin{array}{l}\text { Fax +49 761 4520714 } \\
\text { Information@Karger.de } \\
\text { www.karger.com }\end{array}$ & $\begin{array}{l}\text { Accessible online at: } \\
\text { www.karger.com/onk }\end{array}$
\end{tabular}

Dr. med. Jan Schildmann, M.A.

Institut für Medizinische Ethik und Geschichte der Medizin Ruhr-Universität Bochum

Malakowturm - Markstr. 258a, 44799 Bochum, Germany

Tel. +49 234 32-28654, Fax -14205

jan.schildmann@rub.de 


\section{Hintergrund}

Die Verteilung knapper Ressourcen im Gesundheitswesen ist Gegenstand der aktuellen Diskussion in vielen Ländern Europas. In Deutschland wird Rationierung im Sinne einer Begrenzung wirksamer Interventionen von politischer Seite verneint. Allerdings verweisen sowohl die Vertreter der verfassten Ärzteschaft als auch Wissenschafter unterschiedlicher Disziplinen auf die faktisch bestehende implizite Rationierung im deutschen Gesundheitssystem (siehe Beitrag von D. Strech in diesem Supplement). Der Ansatz einer expliziten Rationierung anhand transparenter Kriterien wird in verschiedenen europäischen Ländern verfolgt. Ein auch in Deutschland vielfach diskutiertes Beispiel ist in diesem Zusammenhang die Begrenzung öffentlich finanzierter Gesundheitsleistungen auf der Grundlage von Kosten-Nutzen-Bewertungen durch das National Institute for Clinical Excellence (NICE) in England [1,2].

Im Gegensatz zum internationalen Forschungsstand [3, 4] liegen für Deutschland nur wenige empirische Untersuchungen zur ärztlichen Wahrnehmung und Bewertung ökonomischer Aspekte in der klinischen Praxis vor. Die in den Bereichen der Kardiologie und Intensivmedizin erhobenen Studiendaten belegen, dass auch Ärzte in Deutschland potenziell nützliche Maßnahmen aus Kostengründen begrenzen [5, 6]. Für das Fachgebiet der Hämatologie und Onkologie liegen nach Kenntnis der Autoren keine vergleichbaren Untersuchungen aus Deutschland vor. Angesichts des demographisch bedingten Anstiegs von Krebserkrankungen sowie der Entwicklung neuer, kostenintensiver Therapieansätze ist absehbar, dass die Ausgaben für die medizinische Versorgung von an Krebs erkrankten Patienten weiter steigen werden [7-9]. Vor diesem Hintergrund ist zu erwarten, dass Strategien zur Kostenbegrenzung auch erhebliche Auswirkungen für das Fachgebiet der Hämatologie und Onkologie haben werden.

In dieser Arbeit werden ausgewählte Ergebnisse zu Wahrnehmungen und Bewertungen von Kostenaspekten in der klinischen Praxis aus der Perspektive von onkologisch tätigen Ärzten in Deutschland und England vorgestellt. Aufgrund des Mangels an empirischen Studien zu diesem Themenfeld in Deutschland wurden für diesen Beitrag Transkripte, die aus einem Forschungsprojekt zur komparativen medizinethischen Analyse der Entscheidungsfindung am Lebensende in Deutschland und England stammen, erneut ausgewertet [10]. Die Interviewpassagen, in denen die Befragten ökonomische Aspekte in der klinischen Praxis thematisierten, wurden einer inhaltsanalytischen Untersuchung unterzogen. Ziel war die Exploration der ärztlichen Perspektive auf ökonomische Aspekte in der klinischen Praxis unter Berücksichtigung folgender, retrospektiv formulierter Fragestellungen:

- Welchen Stellenwert haben ökonomische Erwägungen im klinischen Alltag nach Wahrnehmung der Befragten?

- Wie bewerten die Befragten ökonomische Erwägungen in Bezug auf die ärztliche Handlungspraxis?
Die Ergebnisse werden insbesondere vor dem Hintergrund der unterschiedlichen Strategien zur Rationierung in Deutschland und England (implizite versus explizite Rationierung) diskutiert.

\section{Studienteilnehmer und Methoden}

Die befragten Ärzte arbeiteten in Krankenhäusern bzw. Schwerpunktpraxen mit einem Schwerpunkt im Bereich der Hämatologie und Onkologie (Deutschland) bzw. in Krankenhäusern des National Health Service (NHS) mit Abteilungen für Medical Oncology (England). Ausgehend von einer Konvenienzstichprobe erfolgte die Gewinnung weiterer Interviewpartner mithilfe des «snowball sampling» (Gewinnung neuer Studienteilnehmer auf Empfehlung vorheriger Interviewpartner) bzw. des «purposive sampling» (Auswahl von Studienteilnehmern anhand von für die Fragestellung relevanten Kriterien). Das Forschungsprojekt erhielt von der Ethikkomission der Medizinischen Fakultät der Ruhr-Universität Bochum (AZ 2749-06) bzw. dem South West Multi-Centre Research Ethics Committee (Ref. 06/MRE06/52) positive Voten.

Der für die Forschungsinterviews verwendete Gesprächsleitfaden wurde unter Berücksichtigung relevanter Publikationen [11, 12] zunächst deutschsprachig entwickelt und im Anschluss in die englische Sprache übersetzt. Die auf Tonband aufgezeichneten Interviews wurden wörtlich transkribiert und anonymisiert. Der Fokus der Fragestellung und initialen Auswertung der Untersuchung lag auf der Rekonstruktion der ärztlichen Perspektive auf Behandlungsentscheidungen bei Patienten mit fortgeschrittenen Tumorerkrankungen [10]. Die in diesem Beitrag ausgewerteten Abschnitte der Interviews umfassen spontane oder durch den Interviewer getriggerte Äußerungen der Befragten zu Kosten-Aspekten in der klinischen Praxis.

Im Rahmen der inhaltsanalytischen Auswertung wurden in einem ersten Analyseschritt thematisch ähnliche Interviewabschnitte mit sogenannten «Kodes» belegt. Im Verlauf des ständigen Vergleichs der Interviewtranskripte («constant comparison») und unter Berücksichtigung verschiedener Interpretationen innerhalb der Autorengruppe und weiterer Wissenschafter wurden die Kodes überarbeitet und in übergeordneten «Kategorien» zusammengefasst [13]. Zur Illustration der Kodes beziehungsweise Kategorien (im Ergebnisteil kursiv gedruckt) wurden repräsentative Zitate ausgewählt. Die englischen Zitate wurden vom Erstautor übersetzt. Die Auswertung erfolgte computergestützt mit dem Programm Atlas.ti 6.

\section{Ergebnisse}

\section{Studienteilnehmer}

Es wurden 17 (Deutschland) beziehungsweise 12 (England) Forschungsinterviews mit onkologisch tätigen Ärzten durchgeführt. Tabelle 1 fasst ausgewählte soziodemographische Daten der Studienteilnehmer in Deutschland und England zusammen.

\section{Forschungsinterviews in Deutschland}

Kostenaspekte hinsichtlich der Diagnostik und Therapie von Tumorerkrankungen werden nach Angaben der Befragten insbesondere von Vertretern der Krankenkassen bzw. der Krankenhausverwaltung an die Ärzteschaft herangetragen (zunehmende Thematisierung). Während in keinem Interview ein konkretes Beispiel genannt wird, in dem diagnostische oder therapeutische Maßnahmen aus Kostengründen vorenthalten wurden, wirken sich unterschiedliche Formen der im- 
Tab. 1. Ausgewählte soziodemographische Merkmale der Studienteilnehmer

\begin{tabular}{lcl}
\hline Merkmal & Deutschland & England \\
\hline Männlich & 7 & 9 \\
Weiblich & 10 & 3 \\
Alter & & \\
$\quad$ 30-35 Jahre & 5 & 4 \\
36-50 Jahre & 8 & 4 \\
51-65 Jahre & 4 & 4 \\
Qualifikation & & \\
$\quad$ Facharzt & 9 & 8 \\
$\quad$ Arzt in Weiterbildung & 8 & 4 \\
\hline
\end{tabular}

pliziten Rationierung, wie die Budgetierung bzw. das Fallpauschalensystem, nach Wahrnehmung der Befragten auf die klinische Handlungspraxis aus. Darüber hinaus werden Gewinninteressen von Krankenhausträgern als weiteres Beispiel für den Einfluss ökonomischer Erwägungen auf die klinische Praxis genannt.

Arzt (Ident. EOL D 17): "Sie wissen, dass es für jede Krankheit eine gewisse optimale Verweildauer gibt, und wenn die überschritten wird, dann wird erst mal nichts bezahlt [...] wir arbeiten halt dann nur für 2/3 oder so etwas [...]. Das ist aber nicht eine Sache, die jetzt unsere Verwaltung oder die Kassen betrifft, sondern es ist ein gesellschaftlicher Trend, der uns durch dieses Honorarsystem zum einen und zum anderen durch die privaten Krankenhäuser mehr oder weniger reingeschmuggelt wird.»

Die Krankenversorgung unterscheidet sich nach Wahrnehmung der Befragten in Abhängigkeit vom Versicherungsstatus (Private versus Gesetzliche Krankenversicherung) des Patienten.

Arzt (Ident. EOL D 8): "Ich kann mich jetzt an Patienten erinnern, wo dann z.B. eben die palliative Bestrahlung, das war dann eben eine Sache von 2 Stunden sozusagen. Jetzt würde ich [...] behaupten, dass das die Kassenpatienten auch so kriegen, aber so schnell vielleicht nicht.»

Weiterhin schätzen Vertreter universitärer Kliniken ihre Behandlungsoptionen auch aufgrund der Querverbindungen zwischen klinischer Forschung und Patientenversorgung im Vergleich zu Krankenhäusern mit dem ausschließlichen Auftrag der Krankenversorgung als umfangreicher ein (ungleiches Behandlungsangebot).

Arzt (Ident. EOL D 3): «Ja, letztendlich ist es halt so, dass du hier an der Uni immer die Möglichkeiten von allem hast. Erstens kommst du an Medikamente leichter ran, hast auch mehr Unterstützung durch die Pharmafirma, die auch mal Medikamente, die nicht für die Indikation zugelassen sind, kostenlos zur Verfügung stellen.»

Der Einfluss ökonomischer Erwägungen auf die klinische Praxis wird kritisch und überwiegend negativ bewertet. Im Vordergrund stehen die Sorge vor ungerechten Rationierungskriterien (z.B. altersabhängige Rationierung) und negativen Einflüssen auf die Arzt-Patient-Beziehung.
Arzt (Ident. EOL D 17): «Das Problem ist ja, dass das Verhältnis Arzt-Patient immer ein unangetastetes Zweierverhältnis war und jetzt schiebt sich [...] der Homo oeconomicus dazwischen.»

Allerdings werden vereinzelt die Einbeziehung ökonomischer Überlegungen bei Behandlungsentscheidungen und die Ausbildung gesundheitsökonomischer Kompetenzen auch gefordert.

Arzt (Ident. EOL D 5): "[...] es muss bewusster auch im Studium Kostenaspekte gelehrt werden und antrainiert werden, dass das auch den Ärzten, die heute tätig sind, nicht so mühsam und lästig vorkommt.»

\section{Forschungsinterviews in England}

Im Unterschied zu den befragten Ärzten in Deutschland nennen die onkologisch tätigen Ärzte in England konkrete Situationen, in denen auf potenziell nützliche medizinische Maßnahmen aufgrund von Kostenerwägungen verzichtet wird.

Arzt (Ident. EOL EW 5): "Zum Beispiel, Sunitinib ist hier nicht für Nierenkrebs zugelassen [...]. Ich meine, wenn es unbegrenzte Geldreserven gäbe, dann wäre es zugelassen [...]. Wir haben Glück [...] wir können klinische Studien durchführen, also wenn etwas nicht zugelassen ist, dann haben wir höchstwahrscheinlich eine Studie mit einem ähnlichen Wirkstoff [...] in die wir versuchen, Leute einzuschließen.»

Neben den Vorgaben durch das National Institute für Clinical Excellence (NICE) auf der Makroebene werden Allokationsentscheidungen nach Darstellung der Befragten auch auf der Mesoebene - in den einzelnen Kliniken bzw. Primary Care Trusts - sowie auf der Ebene der individuellen Arzt-PatientBeziehung (Mikroebene)getroffen. Der Einschluss von Patienten in klinische Studien wird von mehreren Befragten als Ausweg genannt, um Patienten mit fortgeschrittenen Tumorerkrankungen noch weitere, nicht durch den National Health Service (NHS) finanzierte Tumortherapien anzubieten.

Arzt (Ident. EOL EW 11): "Ich meine, es ist hier so, dass eine Wunschliste von Medikamenten, die wir wollen, an unsere Beauftragten geht. Und gelegentlich stimmt die Kommission $z u$, es zu finanzieren, auch wenn NICE es nicht in Betracht zieht ...»

Die Einschränkung der durch den NHS finanzierten Maßnahmen wird von den Interviewpartnern unter Verweis auf begrenzt zur Verfügung stehende Mittel im Gesundheitswesen als Notwendigkeit erachtet. Darüber hinaus wird auch die von industriellen Interessen unabhängige Prüfung von Substanzen hinsichtlich deren (Kosten-)Effektivität durch NICE positiv hervorgehoben. Nach Ansicht der Befragten sollten Rationierungsentscheidungen primär auf der Makroebene und nicht auf der Ebene der Arzt-Patient-Beziehung erfolgen.

Arzt (Ident. EOL EW 2): "Wir haben [...] Rationierung durch das National Institute of Clinical Excellence [...]. Es ist oft notwendig, dass es nach den klinischen Studien der Pharmakonzerne eine weitere Evaluation dieser Behandlungen gibt.» 
Neben der Notwendigkeit einer ausreichenden Datengrundlage und fachlichen Expertise für Rationierungsentscheidungen wird von den Befragten die Präferenz für die Zuordnung dieser Entscheidungen auf die Makroebene auch mit der daraus resultierenden Entlastung für die Arzt-PatientBeziehung begründet.

Arzt (Ident. EOL EW 5): "Ich würde es [die Rationierung] wahrscheinlich einer zentralisierten Stelle überlassen. Ich denke, wenn man es dem behandelnden Arzt überlässt: A) Du bist weniger objektiv, äh, und $d u$... da spielt Emotion eine Rolle [...].»B) «... NICE hat Tausende von Patienten und die werden von spezialisierten Gesundheitsökonomen ausgewertet, was wir meiner Meinung nach nicht sind.»

\section{Diskussion}

In dieser Arbeit werden nach Kenntnis der Autoren erstmals qualitative Daten zu Kostenaspekten in der klinischen Praxis aus der Perspektive onkologisch tätiger Ärzte in Deutschland und England vorgestellt. Das gewählte qualitativ-empirische Vorgehen (Leitfadeninterviews, Inhaltsanalyse) ermöglicht die Exploration ärztlicher Wahrnehmungen und Bewertungen sowie die Explikation von informellem Expertenwissen, das für die aktuelle Debatte über Strategien zur Allokation begrenzter Mittel im Gesundheitswesen fruchtbar gemacht werden kann [11]. Mit Blick auf die Dateninterpretation sind verschiedene Limitationen zu bedenken. Dies gilt insbesondere für die fehlende Repräsentativität der Ergebnisse, die in der kleinen Stichprobe, im Fokus der Untersuchung auf Behandlungsentscheidungen bei fortgeschrittenen Tumorerkrankungen sowie in der fehlenden theoretischen Saturierung begründet ist. Weiterhin sind sozial erwünschte Antworten sowie die durch den qualitativen Forschungsansatz bedingten subjektiven Setzungen bei der Dateninterpretation als methodisch bedingte Einschränkungen zu berücksichtigen.

Im Unterschied zu ihren deutschen Kollegen benennen die befragten Ärzte in England konkrete Situationen, in denen potenziell nützliche Interventionen aus Kostengründen nicht eingesetzt werden. Die Ergebnisse quantitativer Untersuchungen auch aus den Bereichen der Kardiologie und Intensivmedizin belegen allerdings, dass Ärzte auch in Deutschland potenziell nützliche Maßnahmen aus Kostengründen nicht durchführen $[4,5]$. Die für unsere Untersuchung gewählte Methode der persönlichen Interviews hat möglicherweise zu einer Verzerrung der Ergebnisse im Sinne sozial erwünschter Antworten geführt. Die Budgetierung und das Fallpauschalensystem als Formen der impliziten Rationierung sowie Gewinninteressen von Klinikträgern werden von den in Deutschland tätigen Ärzten als ökonomische Einflussfaktoren wahrgenommen und nicht zuletzt mit dem Verweis auf mögliche negative Einflüsse auf die Arzt-Patient-Beziehung kritisiert. Dagegen wird eine Begrenzung medizinischer Maßnahmen aus ökonomischen Erwägungen von den Befragten in Eng- land nicht grundsätzlich abgelehnt. Vielmehr wird dies unter Verweis auf begrenzt zur Verfügung stehende Mittel als notwendig erachtet. Die Kosten-Nutzen-Bewertung durch NICE wird weiterhin unter dem Gesichtspunkt einer unabhängigen Prüfung nach der Zulassung eines Arzneimittels befürwortet.

Die Aussagen der Ärzte in beiden Länder können als Ablehnung von Allokationsentscheidungen auf der Mikroebene interpretiert werden. Aus medizinethischer Perspektive wird die Zuordnung von Allokationsentscheidungen auf die Makro- oder Mikroebene kontrovers beurteilt. Die fachliche Expertise des Arztes und seine Kenntnis der Krankengeschichte(n) der Patienten werden als Argumente für die implizite Rationierung auf der Mikroebene angeführt. Dies soll im Einzelfall eine angemessene Entscheidung und die effiziente Verteilung begrenzter Ressourcen ermöglichen (siehe auch Beitrag von E. Winkler in diesem Supplement). Dagegen werden der Anspruch von Patienten, bei vergleichbaren Erkrankungssituationen eine vergleichbare Behandlung zu erhalten, sowie die Forderung nach Transparenz zugunsten von Rationierungsentscheidungen auf der Makroebene angeführt. Mit Blick auf die vielerorts eingeführten finanziellen Anreize zur Einsparung von Mitteln wird weiterhin kritisiert, dass Allokationsentscheidungen auf der Mikroebene den Arzt in die problematische Situation bringen, zwischen der bestmöglichen und der kostengünstigsten Behandlung des Patienten zu entscheiden («double agent») [1,14].

Zusammenfassend bieten die vorliegenden Ergebnisse einen Einblick in die Wahrnehmung und Bewertung ökonomischer Aspekte in der klinischen Praxis aus der Perspektive onkologisch tätiger Ärzten in Deutschland und England. Die unterschiedlichen Strategien der Rationierung (implizit versus explizit) in den Gesundheitswesen der beiden Länder stehen möglicherweise im Zusammenhang mit den als länderspezifisch ermittelten Narrativen. Empirisch-ethische Untersuchungen zur Perspektive von Ärzten, Patienten und weiteren Interessengruppen auf medizinisch und ethisch relevante Aspekte beim Umgang mit begrenzten Mitteln im Gesundheitswesen können die auch in Deutschland zunehmend geführte Diskussion über angemessene Strategien zur Verteilung knapper Ressourcen im Gesundheitswesen informieren.

\section{Disclosure Statement}

Die Autoren geben an, dass keine Interessenkonflikte bestehen.

\section{Danksagung}

Die Autoren danken allen an der Untersuchung teilnehmenden Ärzten für ihre Bereitschaft zur Studienteilnahme sowie Frau Anne Baumann, B.A. und Herrn Johannes Lange, Dipl. Soz. für ihre Anregungen zur Auswertung der Daten. Die Arbeit von Dr. Schildmann wurde mit Mitteln der Heinrich und Alma Vogelsangstiftung der Medizinischen Fakultät, Ruhr-Universität Bochum unterstützt. 


\section{Referenzen}

1 Bundesärztekammer: Stellungnahme der Zentralen Kommission zur Wahrung ethischer Grundsätze in der Medizin und ihren Grenzgebieten (Zentrale Ethikkommission) bei der Bundesärztekammer. Priorisierung medizinischer Leistungen im System der Gesetzlichen Krankenversicherung (GKV). Dtsch Arztebl 2007;104:A2750-A2754.

2 Marckmann G: Priorisierung im Gesundheitswesen: Was können wir aus den internationalen Erfahrungen lernen. Z Evid Fortbild Qual Gesundhwes 2009;103:85-91.

3 Hurst SA, Chandros Hull S, DuVal G, Danis M: Physicians' responses to resource constraints. Arch Intern Med 2005;165:639-644.

4 Strech D, Synofzik M, Marckmann G: How physicians allocate scarce resources at the bedside. A systematic review of qualitative studies. J Med Philos 2008;33:80-99.

5 Boldt J, Schöllhorn T: Rationierung ist längst Realität. Ergebnisse einer Fragebogenaktion auf deutschen Intensivstationen. Dtsch Arztebl 2008;105: A995-A997.
Strech D, Marckmann G: Wird in deutschen Kliniken rationiert oder nicht? Wie genau wir es wissen und warum es nicht die wichtigste Frage sein sollte. Dtsch Med Wochenschr 2010;135:1498-1502.

7 Bach PB: Limits on Medicare's ability to control rising spending on cancer drugs. N Engl J Med 2009;360:626-633.

8 Ludwig WD, Fetscher S, Schildmann J: Teure Innovationen in der Onkologie für alle? Überlegungen $\mathrm{zu}$ Voraussetzungen für rationale Pharmakotherapie und ethische Herausforderungen. Onkologe 2009;15:1004-1014.

9 Robert Koch Institut: Verbreitung von Krebserkrankungen in Deutschland. www.rki.de/cln_178/ nn_204124/sid_677B981E96C280FCDA12616F419 5688E/DE/Content/GBE/DachdokKrebs/Praevalenz/Lokalisationen/Krebslokalisationen_tabelle_ gesamt.html, 2010.
0 Schildmann J, Vollmann J: Behandlungsentscheidungen bei Patienten mit fortgeschrittenen Tumorerkrankungen. Eine empirisch-ethische Untersuchung zur ärztlichen Indikationsstellung und Entscheidungsfindung. Dtsch Med Wochenschr 2010;135:2230-2234.

11 Albisser H, Reiter-Theil S: «Alter» und «Kosten» - Faktoren bei Therapieentscheiden am Lebensende? Eine Analyse informeller Wissensstrukturen bei Ärzten und Pflegenden. Ethik Med 2007;4:103119.

12 Bosshard G, Nilstun T, Bilsen J, et al.; for the European End-of-Life Consortium: Forgoing treatment at the end of life in 6 European countries. Arch Intern Med 2005;165:401-407.

13 Mayring P: Qualitative Inhaltsanalyse. Forum Qualitative Sozialforschung/Forum: Qualitative Social Research (online journal) 2000;1(2). Verfügbar über: www.qualitative-research.net/fqs-texte/2-00/200mayring-d.htm, letzter Zugriff am 07.10.2010.

14 Sulmasy DP: Cancer care, money, and the value of life: Whose justice? Which rationality? J Clin Oncol 2007;25:217-222. 